\title{
Genetic Examination of the PLXNA2 gene in Japanese and Chinese schizophrenics
}

Mizuho Nakajima a,b, Kazuo Yamada a , Eiji Hattori ${ }^{\text {a }}$, Yoshimi Iwayama a , Tomoko Toyota ${ }^{\text {a }}$, Yasuhide Iwata ${ }^{c}$, Kenji J. Tsuchiya ${ }^{c}$, Genichi Sugihara ${ }^{c}$, Kenji Hashimoto ${ }^{d}$, Hiroyuki Watanabe ${ }^{\mathrm{e}}$, Masaomi Iyo ${ }^{\mathrm{e}}$, Mitsuru Kikuchi ${ }^{\mathrm{f}}$, Yuji Okazaki ${ }^{\mathrm{g}}$ Takeo Yoshikawa a,h,*

${ }^{a}$ Laboratory for Molecular Psychiatry, RIKEN Brain Science Institute, Wako, Saitama

$$
\text { 351-0198, Japan }
$$

${ }^{\mathrm{b}}$ Department of Paediatrics, Tokyo Medical University, Tokyo 160-0023, Japan

${ }^{\mathrm{c}}$ Department of Psychiatry and Neurology, Hamamatsu University School of Medicine, Hamamatsu, Shizuoka 431-3192, Japan

${ }^{\mathrm{d}}$ Division of Clinical Neuroscience, Chiba University Center for Forensic Mental Health, Chiba 260-8670, Japan

e Department of Psychiatry, Chiba University Graduate School of Medicine, Chiba 260-8670, Japan

${ }^{\mathrm{f}}$ Department of Psychiatry and Neurobiology, Kanazawa University Graduate School of Medical Science, Kanazawa 920-8641, Japan

g Tokyo Metropolitan Matsuzawa Hospital, Setagaya-ku, Tokyo 156-0057, Japan

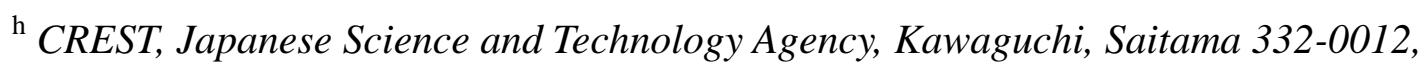
Japan

*Corresponding author:

Takeo Yoshikawa, MD, PhD

Laboratory for Molecular Psychiatry

RIKEN Brain Science Institute

2-1 Hirosawa, Wako-city, Saitama 351-0198, Japan 
Tel: +81(Japan)-48-467-5968

Fax: +81(Japan)-48-467-7462

E-mail: takeo@brain.riken.jp 


\begin{abstract}
(within 250 words)
Aberrant neuronal development is one of the integrative theories for the etiology of schizophrenia. The plexin A2 (PLXNA2) gene is one of the receptor genes for axonal guidance factors. Recently, four single nucleotide polymorphisms (SNPs), rs841865, rs752016, rs1327175 and rs2498028, from the PLXNA2 genomic interval have been reported to be associated with schizophrenia in samples from European Americans, Latin Americans and Asian Americans. We tested these four SNPs for association with disease in two Asian populations: 1140 case-control Japanese samples and 293 Chinese pedigrees (1,163 samples). In the Japanese samples, significant differences in the allelic frequency and genotypic distribution of rs841865 ( $p=0.019$ and 0.020 , respectively) were observed between cases and controls. Haplotype analysis also revealed a significant association of the gene with the disease (global $p=0.028$ ). In contrast, there was no genetic contribution of PLXNA2 to Chinese schizophrenia, either by linkage analysis or association tests (allelic and haplotypic transmission disequilibrium tests). These findings suggest that $P L X N A 2$ confers a varying genetic risk for schizophrenia among different populations.
\end{abstract}

Keywords: plexin A2; chromosome 1q; association; linkage 


\section{Introduction}

Synaptic pathology is a feature of schizophrenia, detectable as altered expression of synaptic proteins and dendritic abnormalities. These changes may be a manifestation of the aberrant neurodevelopment thought to underlie this disorder. The discovery of genes involved in key developmental processes, such as neuronal migration, axon guidance and synaptogenesis, allows this hypothesis to be tested molecularly.

Recently, Mah et al. (2006) identified the plexin A2 gene (PLXNA2) as a candidate for schizophrenia in a whole-genome association study (Mah et al., 2006). Plexins are transmembrane proteins that act as receptors for the semaphorin family of secretion signal proteins. Plexin members have been implicated in the development of axonal projections and neural regeneration (Cheng et al., 2001; Tamagnone et al., 1999; Winberg et al., 1998). Nine different plexins, divided into four subfamilies (A through to D), have been reported: plexins A1-4, B1-3, C1 and D1 (Tamagnone et al., 1999). Plexin A2 heterodimerizes with either neuropilin (NRP) 1 or 2 and forms a receptor for the class 3 semaphorins (sema3), which are secreted axonal chemorepellents (Tamagnone et al., 1999). Interestingly, the plexin A2/NRP 1 ligand, sema3A, was shown to be increased in the cerebellum of patients with schizophrenia (Eastwood et al., 2003). In addition, PLXNA2 is located at chromosome 1q32, a region frequently reported as a susceptibility locus in linkage studies of schizophrenia (Ekelund et al., 2001; Gurling et al., 2001; Hovatta et al., 1999; Hwu et al., 2003).

In this study, we genotyped four PLXNA2 single nucleotide polymorphisms (SNPs): rs841865, rs752016, rs1327175 and rs2498028. These were selected from a pool of SNPs that have shown significant association with schizophrenia in several populations, as reported by Mah et al. (2006), and investigated for association with disease in Japanese and Chinese populations and linkage to disease in the Chinese population. 


\section{Materials and methods}

\subsection{Subjects}

Two cohorts were included in this study: one set of Japanese case-control samples composed of 570 schizophrenic patients (285 men, 285 women; mean age $47.0 \pm 11.4$ years) and 570 age and sex matched controls (285 men, 285 women; mean age $46.7 \pm$ 11.1 years), and a second set of 293 Chinese schizophrenic families (nine trios and 284 quads) composed of 1163 subjects collected by the NIMH initiative (http://nimhgenetics.org/). For the Japanese samples, the diagnosis of schizophrenia was made by consultation according to DSM-IV criteria with consensus from at least two experienced psychiatrists. Control subjects were recruited from hospital staff and volunteers who showed no evidence of present or past psychoses during brief interviews with psychiatrists. The study was approved by the Ethics Committees of RIKEN, Hamamatsu University, Chiba University and Kanazawa University. All participants provided written informed consent.

\subsection{Genotyping}

The SNPs were typed using the TaqMan system (Applied Biosystems, Foster City, CA). Polymerase chain reaction (PCR) was performed using an ABI 9700 thermocycler and fluorescent signals were analyzed using an ABI 7900 sequence detector single point measurement and SDS v2.0 software (Applied Biosystems). Conflicts or flagged alleles were resolved by re-genotyping (the initial success rates of genotyping using the TaqMan system were 99.3\% in Japanese samples and 99.7\% in Chinese samples). Microsatellite marker loci were amplified by PCR using fluorescently labeled primers. PCR fragments were analyzed on an ABI PRISM 3730 Genetic Analyzer (Applied Biosystems). 
Genotypes were determined using GeneScan 3.5.2 and Genotyper 3.6 software (Applied Biosystems).

\subsection{Statistical analyses}

In the Japanese case-control analysis, the allelic and genotypic distributions were tested for association by Fisher's exact test. Haplotypic association analysis was performed using the COCAPHASE program in the UNPHASED package (http://www.rfcgr.mrc.ac.uk/ fdudbrid/software/unphased/) (Dudbridge, 2003). To estimate the degree of linkage disequilibrium (LD) between pairs of loci in Japanese and Chinese populations, the standardized disequilibrium coefficient ( $\left.D^{\prime}\right)$ and the squared correlation coefficient $\left(r^{2}\right)$ were calculated using COCAPHASE. In the analysis of Chinese pedigree samples, transmission disequilibrium test (TDT) analysis of SNPs and haplotypic association analysis were performed using FBAT (http://www.biostat.harvard.edu/ fbat/default.html) software. Linkage analysis was performed using the MERLIN program (http://www.sph.umich.edu/csg/abecasis/Merlin/tour/parametric.html) (Abecasis et al., 2002).

\section{Results}

The SNP information, allelic and genotypic frequencies of each SNP in Japanese schizophrenic patients and controls are summarized in Table 1. Allelic and genotypic distributions of SNP rs841865 differed significantly between cases and controls $(p=$ 0.019 and 0.020 , respectively) (Table 1 ). In haplotype analysis, the haplotype TCCC was over-represented in cases $(p=0.015)$ and haplotype CCCC was over-represented in controls, although the frequency of this haplotype was rare $(p=0.049)$, and the global $p$ 
value was 0.028 (Table 2). LD analysis showed that this region is in strong LD in both Japanese and Chinese populations (Table 3). Meanwhile, no association of PLXNA2 with schizophrenia was found in the TDT and haplotype analyses of the Chinese pedigree samples (Tables 4 and 5). To widen the investigation into the candidate disease locus, we genotyped two additional microsatellite markers, D1S2692 and D1S245 (physical positions 204,427,271 and 205,994,817, respectively in the NCBI Build 35) located on both sides of the SNP cluster, and performed linkage analysis using 278 sib-pairs from the Chinese samples. However, no evidence of linkage was detected in the region (LOD $\leq$ 0.34, Fig. 1).

\section{Discussion}

In this study, we evaluated the role of PLXNA2 in schizophrenia using Japanese and Chinese samples. We detected different genetic associations for the four SNPs (rs841865, rs752016, rs1327175 and rs2498028) between the two Asian cohorts. In the Japanese samples, we observed a nominally significant genetic predisposition of PLXNA2 to schizophrenia through rs841865, although its odds ratio displayed an opposite effect in the disease, compared to a previous report (samples from European Americans, Latin Americans and Asian Americans) (Mah et al., 2006), and this significance disappeared after Bonferroni correction. By contrast, we did not detect any genetic contribution of PLXNA2 to schizophrenia in Chinese samples, either by family-based SNP and haplotype transmission tests or linkage analysis. The exact causes of these discrepancies are unknown, but they may be attributed to statistical fluctuation because of insufficient sample size, differences in the fine structure of LD in different sample populations, genetic and/or allelic heterogeneity and varying effects of the candidate gene among the different ethnic populations. Although there is some data to suggest that unrelated case-control and family-based designs give overall similar estimates of association 
(Evangelou et al., 2006), the different designs may not be fully comparable. Therefore, it would be of interest to test the gene's effects in a case-control study design using Chinese cohorts. It would also be important to re-sequence the area for a detailed examination. Recently, Fujii et al. (2007) reported the failure of association between PLXNA2 and Japanese schizophrenia. Their sample size (304 schizophrenics and 336 controls) was approximately half that of this study. Taken together, the current evidence for the role of PLXNA2 in Japanese cohorts is modest and limited.

In summary, based on the prevailing view that schizophrenia is a disorder of neurodevelopment and synaptic plasticity (Sawa and Snyder, 2002), continued investigations of PLXNA2 are warranted in independent data sets, because the gene product is one of the receptors for axonal guidance factors. The gene is also of interest because its chromosomal locus (1q32) overlaps with a previously reported disease locus.

\section{Acknowledgments}

This work was supported by RIKEN BSI Funds, Research on Brain Science Funds from the Ministry of Health Labor and Welfare, Grant-in Aid from the MEXT and CREST funds from the Japan Science and Technology Agency, Japan. We thank the members of the Research Resource Center at the RIKEN Brain Science Institute for the sequencing and GeneScan typing service.

Acknowledgment for Schizophrenia Biomaterials and Clinical Data (Chinese samples): Data and biomaterials were collected in three projects that participated in the National Institute of Mental Health (NIMH) Schizophrenia Genetics Initiative. From 1991-97, the Principal Investigators and Co-Investigators were: Harvard University, Boston, MA, U01 MH46318, Ming T. Tsuang, M.D., Ph.D., D.Sc., Stephen Faraone, Ph.D., and John Pepple, Ph.D.; Washington University, St. Louis, MO, U01 MH46276, C. Robert Last updated: June 14, 2006-7 Cloninger, M.D., Theodore Reich, M.D., and Dragan Svrakic, 
M.D.; Columbia University, New York, NY U01 MH46289, Charles Kaufmann, M.D., Dolores Malaspina, M.D., and Jill Harkavy Friedman, Ph.D. The data from the Han Chinese Schizophrenia Linkage Study were collected with funding from grant R01 MH59624 from the US National Institute of Mental Health to Ming T. Tsuang, MD, PhD (Principal Investigator). Other participants in the US were Stephen V. Faraone, $\mathrm{PhD}$ (Co-Principal Investigator), Shao Zhu, MD (Project Coordinator) and Xingjia Cui, MD (Project Coordinator). The project leaders in Taiwan were Hai-Gwo Hwu, M.D. (Taiwan Principal Investigator, National Taiwan University Hospital), Wei J. Chen, M.D. Sci.D. (Taiwan Co-Principal Investigator). Other participants in Taiwan were: Chih-Min Liu,M.D., Shih-Kai Liu, M.D., Ming-Hsien Shieh, M.D., Tzung-Jeng Hwang, M.D., MPH, Ming-Ming Tsuang, M.D., Wen Chen OuYang, M.D., Ph.D., Chun-Ying Chen, M.D., Chwen-Cheng Chen, M.D., Ph.D. Jin-Jia Lin,M.D., Frank Huang-Chih Chou, M.D., PH.D., Ching-Mo Chueh, M.D., Wei-Ming Liu, M.D., Chiao-Chicy Chen, M.D. Jia-Jiu Lo, M.D., Jia-Fu Lee, M.D., Ph.D. Seng Shen, M.D., Yung Feng, M.D. Shin-Pin Lin, M.D, Shi-Chin Guo, M.D, Ming-Cheng Kuo, M.D., Liang-Jen Chuo, M.D., Chih-Pin Lu, M.D., Deng-Yi Chen, M.D, Huan-Kwang Ferng, M.D., Nan-Ying Chiu, M.D., Wen-Kun Chen, M.D. Tien-Cheng Lee, M.D. Hsin-Pei Tang, M.D. Yih-Dar Lee, M.D., Wu-Shih Wang, M.D. For-Wey Long, M.D., PhD., Tiao-Lai Huang, M.D., Jung-Kwang Wen, M.D., Cheng-Sheng Chen, M.D., Wen-Hsiang Huang, M.D., Shu-Yu Yang, M.D., Mei-Hua Hall, Cheng-Hsing Chen. M.D. The project leaders in the People' s Republic of China were Xiaogang Chen M.D., PhD (China Principal Investigator, Institute of Mental Heath, Xiang-ya Teaching Hospital, Central South University), and Xingqun Ni, M.D. (Original Principal Investigator, Sun Yat-sen University). Other participants in China were: Liwen Tan, M.D., PhD, Liang Zhou, M.D., PhD, Jiajun Shi, M.D., PhD, Xiaoling He, M.D., PhD, Xiogzhao Zhu, M.D., PhD, Lingjian Li, M.D., PhD, Ming Wang, M.D., Tiansheng Guo, M.D., Xiaqi Shen, M.D., PhD., Jinghua Yang, M.D. ENH/Northwestern University, Evanston, IL, MH059571, Pablo V. Gejman, M.D. 
(Collaboration Coordinator; PI), Alan R. Sanders, M.D.; Emory University School of Medicine, Atlanta, GA,MH59587, Farooq Amin, M.D. (PI); University of California, San Francisco, CA,MH60870, William Byerley, M.D. (PI); University of Iowa, Iowa, IA,MH59566, Raymond Crowe, M.D. (PI), Donald Black, M.D.; Washington University, St. Louis, MO, U01, MH060879, C. Robert Cloninger, M.D. (PI); University of Colorado, Denver, CO, MH059565, Robert Freedman, M.D. (PI), Ann Olincy, M.D.; University of Pennsylvania, Philadelphia, PA, MH061675, Douglas Levinson M.D. (PI), Nancy Buccola APRN, BC, MSN, New Orleans, Louisiana; University of Queensland, Queensland, Australia, MH059588, Bryan Mowry, M.D. (PI); Mt. Sinai School of Medicine, New York, NY,MH59586, Jeremy Silverman, Ph.D. (PI). 


\section{References}

Abecasis, G.R., Cherny, S.S., Cookson, W.O., Cardon, L.R., 2002. Merlin--rapid analysis of dense genetic maps using sparse gene flow trees. Nat Genet 30, 97-101.

Cheng, H.J., Bagri, A., Yaron, A., Stein, E., Pleasure, S.J., Tessier-Lavigne, M., 2001. Plexin-A3 mediates semaphorin signaling and regulates the development of hippocampal axonal projections. Neuron 32, 249-263.

Dudbridge, F., 2003. Pedigree disequilibrium tests for multilocus haplotypes. Genet Epidemiol 25, 115-121.

Eastwood, S.L., Law, A.J., Everall, I.P., Harrison, P.J., 2003. The axonal chemorepellant semaphorin 3A is increased in the cerebellum in schizophrenia and may contribute to its synaptic pathology. Mol Psychiatry 8, 148-155.

Ekelund, J., Hovatta, I., Parker, A., Paunio, T., Varilo, T., Martin, R., Suhonen, J., Ellonen, P., Chan, G., Sinsheimer, J.S., Sobel, E., Juvonen, H., Arajarvi, R., Partonen, T., Suvisaari, J., Lonnqvist, J., Meyer, J., Peltonen, L., 2001. Chromosome 1 loci in Finnish schizophrenia families. Hum Mol Genet 10, 1611-1617.

Evangelou, E., Trikalinos, T.A., Salanti, G., Ioannidis, J.P., 2006. Family-based versus unrelated case-control designs for genetic associations. PLoS Genet 2, e123.

Fujii, T., Iijima, Y., Kondo, H., Shizuno, T., Hori, H., Nakabayashi, T., Arima, K., Saitoh, O., Kunugi, H., 2007. Failure to confirm an association between the PLXNA2 gene and schizophrenia in a Japanese population. Prog Neuropsychopharmacol Biol Psychiatry 9, 873-877.

Gurling, H.M., Kalsi, G., Brynjolfson, J., Sigmundsson, T., Sherrington, R., Mankoo, B. S., Read, T., Murphy, P., Blaveri, E., McQuillin, A., Petursson, H., Curtis, D., 2001. Genomewide genetic linkage analysis confirms the presence of susceptibility loci for schizophrenia, on chromosomes 1q32.2, 5q33.2, and 8p21-22 and provides support for linkage to schizophrenia, on chromosomes 11q23.3-24 and 20q12.1-11.23. Am J 
Hum Genet 68, 661-673.

Hovatta, I., Varilo, T., Suvisaari, J., Terwilliger, J. D., Ollikainen, V., Arajarvi, R., Juvonen, H., Kokko-Sahin, M. L., Vaisanen, L., Mannila, H., Lonnqvist, J., Peltonen, L., 1999. A genomewide screen for schizophrenia genes in an isolated Finnish subpopulation, suggesting multiple susceptibility loci. Am J Hum Genet 65, 1114-1124.

Hwu, H.G., Liu, C.M., Fann, C.S., Ou-Yang, W.C., Lee, S.F., 2003. Linkage of schizophrenia with chromosome 1q loci in Taiwanese families. Mol Psychiatry 8, 445-452.

Mah, S., Nelson, M.R., Delisi, L.E., Reneland, R.H., Markward, N., James, M.R., Nyholt, D.R., Hayward, N., Handoko, H., Mowry, B., Kammerer, S., Braun, A., 2006. Identification of the semaphorin receptor PLXNA2 as a candidate for susceptibility to schizophrenia. Mol Psychiatry 11, 471-478.

Sawa, A., Snyder, S.H., 2002. Schizophrenia: diverse approaches to a complex disease. Science 296, 692-695.

Tamagnone, L., Artigiani, S., Chen, H., He, Z., Ming, G.I., Song, H., Chedotal, A., Winberg, M.L., Goodman, C.S., Poo, M., Tessier-Lavigne, M., Comoglio, P.M., 1999. Plexins are a large family of receptors for transmembrane, secreted, and GPI-anchored semaphorins in vertebrates. Cell 99, 71-80.

Winberg, M.L., Noordermeer, J.N., Tamagnone, L., Comoglio, P.M., Spriggs, M.K., Tessier-Lavigne, M., Goodman, C.S., 1998. Plexin A is a neuronal semaphorin receptor that controls axon guidance. Cell 95, 903-916. 


\section{Figure legends}

Fig. 1. Linkage analysis of PLXNA2 in Chinese schizophrenia samples.

Linkage analysis was performed using the MERLIN program

(http://www.sph.umich.edu/csg/abecasis/Merlin/tour/parametric.html). 
Table 1

Association analysis of PLXNA2 in Japanese case-control samples

\begin{tabular}{|c|c|c|c|c|c|c|c|c|c|}
\hline \multirow{2}{*}{$\begin{array}{c}\text { dbSNP ID (Location in gene) } \\
\quad\left(\text { Physical position }{ }^{\mathrm{a}}\right)\end{array}$} & & \multicolumn{2}{|c|}{ Allele frequency } & \multirow[t]{2}{*}{$p$ value $^{b}$} & \multirow{2}{*}{$\begin{array}{c}\mathrm{OR}^{\mathrm{c}} \\
(95 \% \mathrm{Cl})\end{array}$} & \multicolumn{3}{|c|}{ Genotype frequency } & \multirow[t]{2}{*}{$p$ value $^{b}$} \\
\hline & & $\mathrm{C}$ & $\mathrm{T}$ & & & $\mathrm{CC}$ & CT & TT & \\
\hline rs841865 (IVS14-101) & Sc & 0.67 & 0.33 & \multirow{3}{*}{0.019} & \multirow{3}{*}{$\begin{array}{c}0.80 \\
(0.67-0.96)\end{array}$} & 0.44 & 0.46 & 0.10 & \multirow{3}{*}{0.020} \\
\hline \multirow[t]{2}{*}{$(204,614,304)$} & $\mathrm{Ct}$ & 0.72 & 0.28 & & & 0.53 & 0.39 & 0.09 & \\
\hline & & $\mathrm{T}$ & C & & & TT & TC & $\mathrm{CC}$ & \\
\hline rs752016 (IVS12-3) & Sc & 0.54 & 0.46 & \multirow{2}{*}{0.422} & \multirow{2}{*}{$\begin{array}{c}0.93 \\
(0.79-1.10)\end{array}$} & 0.29 & 0.51 & 0.21 & \multirow{2}{*}{0.663} \\
\hline \multirow[t]{2}{*}{$(204,626,072)$} & $\mathrm{Ct}$ & 0.56 & 0.44 & & & 0.31 & 0.49 & 0.20 & \\
\hline & & C & G & & & $\mathrm{CC}$ & CG & GG & \\
\hline rs1327175 (IVS12+5) & Sc & 0.91 & 0.09 & \multirow{3}{*}{0.268} & \multirow{3}{*}{$\begin{array}{c}1.18 \\
(0.89-1.54)\end{array}$} & 0.82 & 0.17 & 0.01 & \multirow{3}{*}{0.529} \\
\hline \multirow[t]{2}{*}{$(204,635,529)$} & $\mathrm{Ct}$ & 0.89 & 0.11 & & & 0.79 & 0.19 & 0.01 & \\
\hline & & $\mathrm{T}$ & C & & & TT & TC & $\mathrm{CC}$ & \\
\hline rs2498028 (IVS11+444) & Sc & 0.64 & 0.36 & \multirow{2}{*}{0.185} & \multirow{2}{*}{$\begin{array}{c}0.89 \\
(0.75-1.05)\end{array}$} & 0.39 & 0.49 & 0.11 & \multirow{2}{*}{0.223} \\
\hline$(204,643,708)$ & $\mathrm{Ct}$ & 0.67 & 0.33 & & & 0.44 & 0.45 & 0.11 & \\
\hline
\end{tabular}

a NCBI Build 35 (http://www.ncbi.nlm.nih.gov/)

${ }^{\mathrm{b}} P$ values of $<0.05$ are shown in bold type.

${ }^{\mathrm{c}}$ Odds ratios are calculated using the major allele frequencies.

Sc, schizophrenia; Ct, control 
Table 2

Haplotype analysis of PLXNA2 in Japanese samples

\begin{tabular}{ccccc}
\hline & \multicolumn{2}{c}{ Frequency } & & \\
Haplotypes $^{\mathrm{a}}$ & Sc & Ct & $\chi^{2}$ & $p$ value \\
& 0.54 & 0.56 & 0.484 & 0.487 \\
CTCT & 0.32 & 0.27 & 5.875 & $\mathbf{0 . 0 1 5}$ \\
TCCC & 0.10 & 0.11 & 1.177 & 0.278 \\
CCGT & 0.04 & 0.06 & 3.861 & $\mathbf{0 . 0 4 9}$ \\
CCCC & & & Global $p$ value & $\mathbf{0 . 0 2 8}$ \\
\hline
\end{tabular}

a Haplotypes were constructed using rs841865-rs752016-1327175rs2498028. Minor haplotypes (<0.01) are omitted.

${ }^{\mathrm{b}} P$ values of $<0.05$ are in bold type.

Sc, schizophrenia; Ct, control 
Table 3

LD analysis of four SNPs of PLXNA2 in Japanese (Chinese) samples

\begin{tabular}{|c|c|c|c|c|}
\hline dbSNP ID & rs841865 & rs752016 & rs1327175 & rs2498028 \\
\hline rs841865 & & $\begin{array}{c}1.000 \\
(0.951)\end{array}$ & $\begin{array}{c}1.000 \\
(1.000)\end{array}$ & $\begin{array}{c}0.940 \\
(0.923)\end{array}$ \\
\hline rs752016 & $\begin{array}{c}0.469 \\
(0.491)\end{array}$ & & $\begin{array}{c}1.000 \\
(0.974)\end{array}$ & $\begin{array}{c}1.000 \\
(0.985)\end{array}$ \\
\hline rs1327175 & $\begin{array}{c}0.048 \\
(0.041)\end{array}$ & $\begin{array}{c}0.154 \\
(0.124)\end{array}$ & & $\begin{array}{c}1.000 \\
(1.000)\end{array}$ \\
\hline rs2498028 & $\begin{array}{c}0.695 \\
(0.693)\end{array}$ & $\begin{array}{c}0.619 \\
(0.649)\end{array}$ & $\begin{array}{c}0.061 \\
(0.052)\end{array}$ & \\
\hline
\end{tabular}

Upper right diagonal represents $D^{\prime}$ values and lower left, $r^{2}$ values. Values of $D^{\prime}>0.85$

and $r^{2}>0.1$ are indicated in bold type and in grey cells. For the Chinese population,

only parent samples (deemed to be unrelated to each other) were used for calculation. 
Table 4

TDT analysis of $P L X N A 2$ in Chinese pedigrees

\begin{tabular}{ccc}
\hline dbSNP ID & Z score & $p$ value \\
\hline rs841865 & 0.047 & 0.963 \\
rs752016 & 0.663 & 0.527 \\
rs1327175 & 0.445 & 0.657 \\
rs2498028 & 0.133 & 0.894 \\
\hline
\end{tabular}

Analysis was performed using the FBAT program

(http://www.biostat.harvard.edu/ fbat/default.html). 
Table 5

Haplotype analysis of PLXNA2 in Chinese pedigrees

\begin{tabular}{cccc}
\hline Haplotypes $^{a}$ & Frequency & Z score & $p$ value \\
\hline CTCT & 0.56 & 0.856 & 0.392 \\
TCCC & 0.28 & 0.094 & 0.925 \\
CCGT & 0.09 & -0.523 & 0.601 \\
CCCC & 0.05 & -0.861 & 0.389 \\
\hline
\end{tabular}

Analysis was performed using the FBAT program.

a Haplotype was constructed by rs841865-rs752016-rs1327175-

rs2498028. Minor haplotypes (<0.01) were omitted. 
Figure 1

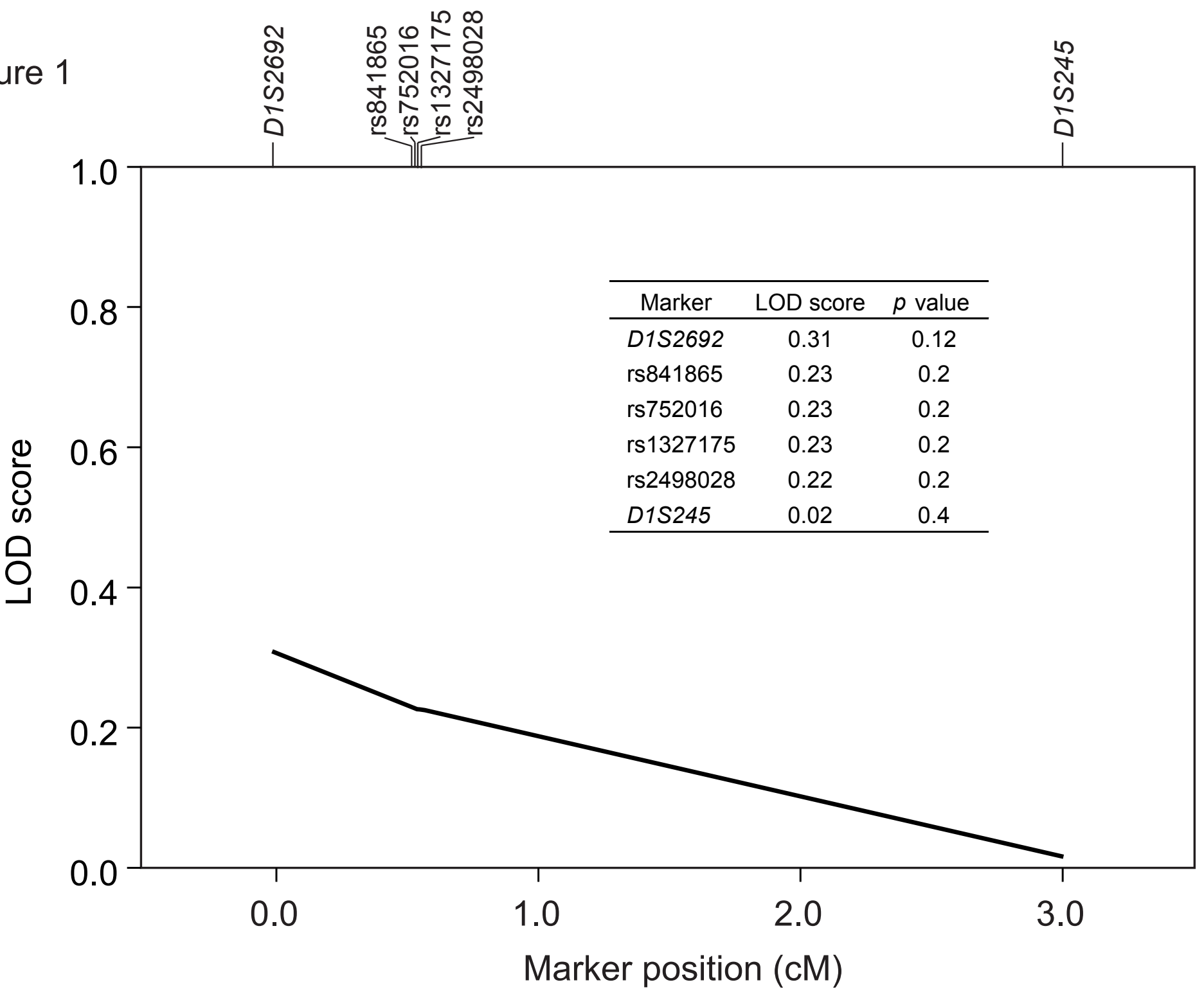




\section{Acknowledgments}

This work was supported by RIKEN BSI Funds, Research on Brain Science Funds from the Ministry of Health Labor and Welfare, Grant-in Aid from the MEXT and CREST funds from the Japan Science and Technology Agency, Japan. We thank the members of the Research Resource Center at the RIKEN Brain Science Institute for the sequencing and GeneScan typing service.

Acknowledgment for Schizophrenia Biomaterials and Clinical Data (Chinese samples): Data and biomaterials were collected in three projects that participated in the National Institute of Mental Health (NIMH) Schizophrenia Genetics Initiative. From 1991-97, the Principal Investigators and Co-Investigators were: Harvard University, Boston, MA, U01 MH46318, Ming T. Tsuang, M.D., Ph.D., D.Sc., Stephen Faraone, Ph.D., and John Pepple, Ph.D.; Washington University, St. Louis, MO, U01 MH46276, C. Robert Last updated: June 14, 2006-7 Cloninger, M.D., Theodore Reich, M.D., and Dragan Svrakic, M.D.; Columbia University, New York, NY U01 MH46289, Charles Kaufmann, M.D., Dolores Malaspina, M.D., and Jill Harkavy Friedman, Ph.D. The data from the Han Chinese Schizophrenia Linkage Study were collected with funding from grant R01 MH59624 from the US National Institute of Mental Health to Ming T. Tsuang, MD, PhD (Principal Investigator). Other participants in the US were Stephen V. Faraone, PhD (Co-Principal Investigator), Shao Zhu, MD (Project Coordinator) and Xingjia Cui, MD (Project Coordinator). The project leaders in Taiwan were Hai-Gwo Hwu, M.D. (Taiwan Principal Investigator, National Taiwan University Hospital), Wei J. Chen, M.D. Sci.D. (Taiwan Co-Principal Investigator). Other participants in Taiwan were: Chih-Min Liu,M.D., Shih-Kai Liu, M.D., Ming-Hsien Shieh, M.D., Tzung-Jeng Hwang, M.D., MPH, Ming-Ming Tsuang, M.D., Wen Chen OuYang, M.D., Ph.D., Chun-Ying Chen, M.D., Chwen-Cheng Chen, M.D., Ph.D. Jin-Jia Lin,M.D., Frank Huang-Chih Chou, M.D., PH.D., Ching-Mo Chueh, M.D., Wei-Ming Liu, M.D., Chiao-Chicy Chen, M.D. Jia-Jiu Lo, M.D., Jia-Fu Lee, M.D., Ph.D. Seng Shen, M.D., Yung Feng, M.D. Shin-Pin Lin, M.D, Shi-Chin Guo, M.D, Ming-Cheng Kuo, M.D., Liang-Jen Chuo, M.D., Chih-Pin Lu, M.D., Deng-Yi Chen, M.D, Huan-Kwang Ferng, M.D., Nan-Ying Chiu, M.D., Wen-Kun Chen, M.D. Tien-Cheng Lee, M.D. Hsin-Pei Tang, M.D. Yih-Dar Lee, M.D., Wu-Shih Wang, M.D. For-Wey Long, M.D., PhD., Tiao-Lai Huang, M.D., Jung-Kwang Wen, M.D., Cheng-Sheng Chen, M.D., Wen-Hsiang Huang, M.D., Shu-Yu 
Yang, M.D., Mei-Hua Hall, Cheng-Hsing Chen. M.D. The project leaders in the People's Republic of China were Xiaogang Chen M.D., PhD (China Principal Investigator, Institute of Mental Heath, Xiang-ya Teaching Hospital, Central South University), and Xingqun Ni, M.D. (Original Principal Investigator, Sun Yat-sen University). Other participants in China were: Liwen Tan, M.D., PhD, Liang Zhou, M.D., PhD, Jiajun Shi, M.D., PhD, Xiaoling He, M.D., PhD, Xiogzhao Zhu, M.D., PhD, Lingjian Li, M.D., PhD, Ming Wang, M.D., Tiansheng Guo, M.D., Xiaqi Shen, M.D., PhD., Jinghua Yang, M.D. ENH/Northwestern University, Evanston, IL, MH059571, Pablo V. Gejman, M.D. (Collaboration Coordinator; PI), Alan R. Sanders, M.D.; Emory University School of Medicine, Atlanta, GA,MH59587, Farooq Amin, M.D. (PI); University of California, San Francisco, CA,MH60870, William Byerley, M.D. (PI); University of Iowa, Iowa, IA,MH59566, Raymond Crowe, M.D. (PI), Donald Black, M.D.; Washington University, St. Louis, MO, U01, MH060879, C. Robert Cloninger, M.D. (PI); University of Colorado, Denver, CO, MH059565, Robert Freedman, M.D. (PI), Ann Olincy, M.D.; University of Pennsylvania, Philadelphia, PA, MH061675, Douglas Levinson M.D. (PI), Nancy Buccola APRN, BC, MSN, New Orleans, Louisiana; University of Queensland, Queensland, Australia, MH059588, Bryan Mowry, M.D. (PI); Mt. Sinai School of Medicine, New York, NY,MH59586, Jeremy Silverman, Ph.D. (PI). 